\title{
FATE: A Polynomial Time Framework for Flow Allocation in MPLS-TE Networks
}

\author{
Samer Lahoud, Géraldine Texier and Laurent Toutain \\ GET/ENST Bretagne \\ Département Réseaux et Services Multimédia \\ 2 rue de la Châtaigneraie - CS 17607 - 35576 Cesson Sévigné Cedex - France \\ samer.lahoud@enst-bretagne.fr, geraldine.texier@enst-bretagne.fr, laurent.toutain@irisa.fr
}

\begin{abstract}
In this paper, we present a novel polynomial time framework for the off-line flow allocation problem in MPLSTraffic Engineering (MPLS-TE) networks. This framework, named FATE (Flow Allocation for Traffic Engineering), encompasses different objectives that illustrate the operator needs for traffic engineering and provides advanced control over the quality of the solution. Typically, we consider minimizing congestion, bandwidth consumption and operational costs. FATE is based on linear programming and presents an efficient solution for the problem both in terms of computational complexity and approximation guarantees. Simulation results show that we can get an efficient trade-off between congestion and bandwidth consumption while having a reduced number of Label Switched Paths (LSPs) for various known topologies.
\end{abstract}

\section{INTRODUCTION}

Traffic engineering is a complex process that includes the set of strategies deployed by network operators in order to get maximum return on network capital expenditures (capex) while reducing operational expenditures (opex). Capex is determined in the network design process when capacity is installed in the core links, whereas opex is related to the cost of managing, supervising and tuning the network in order to get optimal performance. Traffic engineering can be implemented as an on-line and/or an off-line process. Off-line traffic engineering tends to optimize the network considering full knowledge of the demands. This is usually expressed as a traffic demand matrix, with point-to-point demands.

In this work, we introduce a novel polynomial time framework, named FATE (Flow Allocation for Traffic Engineering), for the off-line flow allocation problem in MPLS-Traffic Engineering (MPLS-TE) networks. First, FATE considers minimizing the global network congestion. Second, FATE tries to minimize resource consumption, expressed in terms of the total used bandwidth. Particularly, FATE enables to get efficient return on network capex involved in capacity installation by tuning the relevancy of the first two objectives. Finally, FATE provides a flow allocation solution with a minimum number of Label Switched Paths (LSPs) inducing minimum operational costs for an MPLS-TE network.

This paper is organized as follows: in section II, we introduce relevant related work. In section III, we present the different components in the FATE framework. Then, in section IV, we formulate the mathematical problems used in FATE and emphasize the polynomial time complexity. In section $\mathrm{V}$ we present relevant simulation results. Finally, we conclude by presenting future work and possible extensions to the framework.

\section{RELATED WORK}

Flow allocation is one of the problems that are well studied in the network flow domain. [1] presents a good overview of the general problem, including different formulations and numerous techniques associated with Linear Programs (LPs) such as relaxations and problem decomposition. Moreover, many articles have studied the application to MPLS traffic engineering. In [2], a multi-objective approach to the problem is introduced. This approach considers the compromise between load balancing, network costs, and the number of active LSPs. The problem is formulated as a single objective Mixed Integer LP (MILP) using a weighted sum of the different objectives and solved by means of standard commercial tools. However, no heuristic is presented in order to reduce the computation time. [3] presents a traffic engineering solution for differentiated reliability services. A single weighted objective is used to combine different optimization criteria such as resource consumption, protection level and rejection. The problem is also formulated as a MILP and the authors present two heuristics: a first heuristic is based on successive approximations by continuous relaxations of the MILP and a second heuristic based on the simulated allocation technique. [4] introduces a multi-objective framework based on utilization and resource usage. The authors propose linear programs that find near optimal paths satisfying the given traffic demand under constraints such as maximum hop count, and preferred node/link list. Moreover, a MILP formulation calculates the traffic split ratio for the multiple paths. However, the size of the problems that can be solved is very limited in practice. [5] introduces a novel heuristic for multi-objective traffic engineering in the Virtual Private Network (VPN) context. A multi-stage approach is used in order to successively reduce congestion and bandwidth utilization. Finally, the authors in [6] introduce a simple polynomial time framework for multipath routing. They present efficient techniques that reduce the path set for simple separated problems such as minimum congestion or minimum bandwidth utilization. The mathematical tools introduced in [6] are similar to those used in our work. However, we introduce a more general framework adapted for 


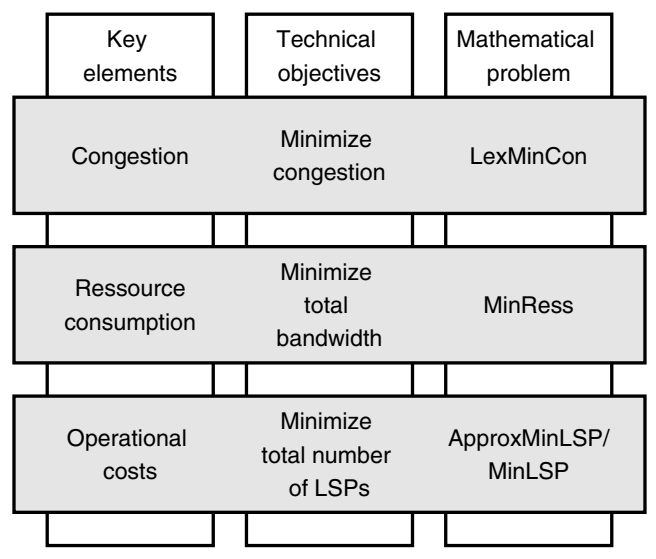

Fig. 1. FATE framework

MPLS traffic engineering. We add a more general concept for minimizing congestion, notably with LexMinCon. Moreover, we emphasize the trade-offs between the different elements in our framework, and provide insights on the tuning process that enables to reach a desired flow allocation solution.

\section{FRAMEWORK PRESENTATION AND MOTIVATION}

Our main contribution in this article is the introduction of a polynomial time framework for flow allocation in MPLSTE networks. This framework, named FATE, is illustrated in Figure 1.

The first key element in the FATE framework is congestion. The corresponding technical objective that illustrates the operator need is to minimize the network congestion. Generally, congestion implies service degradation, thus this objective is well suited for next generation networks that promise to provide quality of service. Moreover, a flow allocation solution with minimal congestion tolerates more dynamic traffic changes, thus the flow reallocation process can be done less frequently. Our approach, formulated in LexMinCon (section IV-A), leads to a network with minimum global congestion (i.e. all link utilization are reduced to their minimal values). Particularly, this is achieved in the search for a lexicographical minimal congestion solution.

However, approaches based solely on network congestion minimization will lead to high resource consumption. Roughly speaking, minimizing congestion will spread the load over the network links. Demands will take relatively longer paths in order to distribute the load, thus will consume more bandwidth on links. Therefore, the second key element in our framework is resource consumption and the corresponding technical objective is to minimize the total used bandwidth formulated in MinRess (section IV-B).

Considering the first two objectives, FATE enables to easily tune their respective relevancy. Particularly, it enables to approximate the optimal congestion in order to get significant reduction in resource consumption.

Finally, the third key element in our framework is operational costs. When there is more than one LSP between two edge Label Switching Routers (LSRs), it may be necessary to perform complex traffic splitting procedures. Moreover, signaling and measurement become more complex when the overlay involves a big number of LSPs. Therefore, our third and final technical objective is to minimize the operational costs for a given solution expressed in terms of the number of LSPs in use. Particularly, ApproxMinLSP (section IV-C) provides an approximation for the minimal number of LSPs. Note that an optimal solution corresponds to an unsplittable multi-commodity flow and is NP-complete [7]. Moreover, an extension is provided in order to get the minimal number of LSPs in MinLSP (section IV-C) via tuning network congestion.

\section{FRAMEWORK COMPONENTS}

In this section, we introduce the mathematical formulation and focus on the interaction between the different framework components. We also give insights on the polynomial time performance of the solution. In the following, we consider an undirected network $\mathcal{G}=(\mathcal{V}, \mathcal{E})$ with $\mathcal{V}$ being the set vertices and $\mathcal{E}$ the set of edges. $\mathcal{V}$ and $\mathcal{E}$ have a respective cardinality of $n$ and $m$. The capacity for each $e \in \mathcal{E}$ is denoted by $c_{e}$. Moreover, we are given a set of LSP demands denoted by $\mathcal{D}$ having a cardinality of $l$. Each demand $d \in \mathcal{D}$ is defined by a triple $\left(s_{d}, t_{d}, h_{d}\right)$, where $s_{i}$ is the source node or ingress LSR, $t_{i}$ the destination node or egress LSR and $h_{d}$ the demand volume or bandwidth request from $s_{d}$ to $t_{d}$. Note that we are using the terms node/vertex/LSR, link/edge interchangeably in this paper.

\section{A. Congestion}

The first objective in FATE is to minimize the global network congestion. This objective is captured in the LexMinCon problem formulated in LP 1. In this formulation, $\operatorname{In}(v)$ (resp.

LP 1 LexMinCon

$$
\text { Lexicographically Minimize } \mathbb{K}
$$

Subject to:

$$
\begin{aligned}
& \sum_{d \in \mathcal{D}} f_{d e} \leq k_{e} c_{e}, \quad \forall e \in \mathcal{E} \\
& \sum_{e \in O u t(v)} f_{d e}-\sum_{e \in \operatorname{In}(v)} f_{d e}=\epsilon_{d v}, \quad \forall(d, v) \in \mathcal{D} \times \mathcal{V} \\
& \epsilon_{d v}=\left\{\begin{array}{ll}
h_{d}, & v=s_{d} \\
-h_{d}, & v=t_{d} \\
0, & v \neq\left(s_{d}, t_{d}\right)
\end{array}\right\}, \forall(d, v) \in \mathcal{D} \times \mathcal{V} \\
& f_{d e} \geq 0, \quad \forall(d, e) \in \mathcal{D} \times \mathcal{E} \\
& k_{e} \geq 0, \forall e \in \mathcal{E}
\end{aligned}
$$

Out $(v)$ ) denote the set of links going in (resp. out of) node $v, f_{d e}$ denotes the flow from demand $d$ routed on link $e$ and $k_{e}$ the congestion on link $e$. In other terms, $k_{e}$ denotes the ratio between the total bandwidth used by flows traversing link $e$ and the capacity of the link. Moreover, we collect the $k_{e}$ congestion values $(\forall e \in \mathcal{E}$ ) and define the congestion vector $\mathbb{K}=\left(k_{1}, k_{2}, \ldots, k_{m}\right)$. Technically, the problem consists of finding a lexicographically minimal congestion vector as 
defined in Definition 1 while satisfying capacity constraints in (2) for each link and flow conservation constraints in (3). Note that LexMinCon is not a mathematical problem since the objective is not a simple maximization or minimization. However, it can be solved in a polynomial time as a sequence of mathematical problems as detailed in the Appendix.

Definition 1 A congestion vector $\mathbb{K}=\left(k_{1}, k_{2}, \ldots, k_{m}\right)$ with elements sorted in a non-increasing order is said to be lexicographically minimal if, for every other congestion vector $\mathbb{K}^{\prime}=$ $\left(k_{1}^{\prime}, k_{2}^{\prime}, \ldots, k_{m}^{\prime}\right)$ with elements sorted in a non-increasing order, an index $0 \leq i \leq m$ exists, such that $k_{j}=k_{j}^{\prime}$ for $0 \leq j \leq i$ and $k_{l} \leq k_{l}^{\prime}$ for $i \leq l \leq m$.

\section{B. Resource Consumption}

The second technical objective in FATE is to minimize the total used bandwidth. The corresponding mathematical problem, MinRess, is formulated in LP 2 as an LP with node-link variables ${ }^{1}$. The size of this LP is polynomial and can be solved in a polynomial time as a multicommmodity network flow problem [8]. In this formulation, we introduce a new congestion vector $\mathbb{K}^{\prime}=\left(k_{1}^{\prime}, k_{2}^{\prime}, \ldots, k_{m}^{\prime}\right)$ given by $\mathbb{K}^{\prime}=\alpha \mathbb{K}$, where $\mathbb{K}$ is the output vector of LexMinCon. Hence, $k_{e}^{\prime}=\alpha k_{e}$ corresponds to the maximum allowed congestion on link $e$. The choice of $\alpha$ determines the interaction between the first two components. It enables to approximate the optimal minimal congestion in order to get significant reduction in resource consumption. Particularly, increasing $\alpha$ enables more efficient resource consumption at the expense of increased network congestion and vice versa. Finally, MinRess provides link flow values for a given congestion state given by $\mathbb{K}^{\prime}$, with minimum bandwidth utilization. These link flow values will be used as an input for the third component that deals with network operational costs.

LP 2 MinRess

$$
\text { Minimize } \sum_{d \in \mathcal{D}} \sum_{e \in E} f_{d e}
$$

Subject to:

$$
\begin{gathered}
\sum_{d \in \mathcal{D}} f_{d e} \leq k_{e}^{\prime} c_{e}, \forall e \in \mathcal{E} \\
\sum_{e \in \operatorname{Out}(v)} f_{d e}-\sum_{e \in \operatorname{In}(v)} f_{d e}=\epsilon_{d v}, \quad \forall d \in \mathcal{D} \\
f_{d e} \geq 0, \quad \forall(d, e) \in \mathcal{D} \times \mathcal{E} \\
\mathbb{K}^{\prime}=\alpha \mathbb{K}, \text { where } \mathbb{K} \text { is the output of LexMinCon }
\end{gathered}
$$

\section{Operational Costs}

The final technical objective in FATE is to minimize the operational costs for a given solution expressed in terms

\footnotetext{
${ }^{1}$ MinRess can be formulated with path-link variables using a pre-calculated set of all possible paths. However, this path set may be exponentially large and, even with column generation techniques, the corresponding LP cannot be solved in a polynomial time.
}

of the number of LSPs in use. Particularly, our framework provides an approximation for the minimal number of LSPs in ApproxMinLSP. Moreover, an optional extension is provided in order to tune the number of LSPs in MinLSP via changing the network congestion state.

First we should point out that the output of the MinRess component is a set of link flow values that represent a network congestion state with minimum bandwidth utilization. This will not give us insights on the LSPs or the paths that demands are actually allocated to. Therefore, we use a standard flow decomposition algorithm [9] in order to get a set of paths with corresponding bandwidth values that saturate link flow values obtained from the MinRess problem. Then, the obtained set of paths denoted $\mathcal{P}_{d}$ is used in the ApproxMinLSP problem. Note that after flow decomposition, the maximal number of paths per demand is equal to $m$ (this is a straight conclusion from the flow decomposition algorithm). Technically, ApproxMinLSP, introduced in LP 3, is a path-link formulation of the MinRess problem. This formulation assigns a variable $f_{d p}$ for the flow routed on path $p$ for demand $d$ and uses a constant denoted by $\delta_{\text {edp }}$ (12) indicating whether path $p$ of the set $\mathcal{P}_{d}$ corresponding to demand $d$ uses link $e$.

$$
\delta_{e d p}=\left\{\begin{array}{ll}
0 & \text { if path } p \in \mathcal{P}_{d} \text { uses link } e \\
1 & \text { elsewhere }
\end{array}\right\}
$$

With ApproxMinLSP, we get the same congestion (given by $\mathbb{K}^{\prime}=\alpha \mathbb{K}$ ) and resource consumption values as the output of the MinRess problem (since we are using the same set of paths $\mathcal{P}_{d}$ as an input), but with a reduced set of paths. The main idea behind reducing the number of used LSPs resides in finding a Basic Feasible Solution (BFS) for the ApproxMinLSP problem. Particularly, in a BFS, the number of non-zero variables is smaller than the number of constraints. Therefore, we get:

$$
\mathrm{Nb} \text { of LSPs } \leq l+m
$$

Hence, ApproxMinLSP provides a $\left(1+\frac{m}{l}\right)$-approximation for the single path solution. Recall that an optimal solution corresponds to an unsplittable multi-commodity flow and is proved to be NP-complete [7]. Particularly, we can get a

LP 3 ApproxMinLSP

$$
\text { Minimize } \sum_{d \in \mathcal{D}} \sum_{p \in \mathcal{P}_{d}} f_{d p}
$$

Subject to:

$$
\begin{gathered}
\sum_{d \in \mathcal{D}} \sum_{p \in \mathcal{P}_{d}} \delta_{e d p} f_{d p} \leq k_{e}^{\prime} c_{e}, \quad \forall e \in \mathcal{E} \\
\sum_{p \in \mathcal{P}_{d}} f_{d p}=h_{d}, \quad \forall d \in \mathcal{D} \\
f_{d p} \geq 0, \quad \forall(d, p) \in \mathcal{D} \times \mathcal{P}_{d}
\end{gathered}
$$

BFS for this problem in a polynomial time using for instance Tardos's algorithm [8]. Moreover, according to Definition 2, the output of ApproxMinLSP in the FATE framework is an $\left(\alpha, 1+\frac{m}{l}\right)$-approximation for the flow allocation problem. 
Definition 2 A flow allocation solution is an $(\lambda, \mu)$ approximation if the corresponding congestion is $\lambda \mathbb{K}$ with $\mathbb{K}$ being the optimal minimal congestion vector, and if the solution uses at most $\mu$ times the number of paths in the optimal solution.

Finally, following the technique used in ApproxMinLSP, we introduce $\operatorname{Min} L S P$, a final optional component that enables to tune the operational costs. MinLSP is formulated in LP 4 as an Mixed Integer LP (MILP) with path-link variables and uses the output path set $\mathcal{P}_{d}^{\prime}$ of ApproxMinLSP. This formulation introduces integer variables $z_{d p}$ that denote whether path $p$ of the set $\mathcal{P}_{d}^{\prime}$ is used by demand $d$ i.e. $z_{d p}=1$ iff $f_{d p}>$ 0 . The latter expression is depicted by constraint (24) that uses the constant $H$, an upper bound on the demand volume $\left(h_{d}<H, \forall d \in \mathcal{D}\right.$ ). Therefore, the objective (21) minimizes the number of LSPs in use. Moreover, MinLSP introduces a final congestion vector $\mathbb{K}^{\prime \prime}$, given by (18), that enables to tune congestion in order to get a reduction in the number of LSPs.

$$
\mathbb{K}^{\prime \prime}=\beta \mathbb{K}^{\prime}
$$

Increasing $\beta$ enables to reduce the number of LSPs at the expense of increased network congestion and vice versa. Note that $\mathbb{K}^{\prime}$ is the congestion vector used in MinRess (given by $\left.\mathbb{K}^{\prime}=\alpha \mathbb{K}\right)$. Technically, in order to explain the functionality of MinLSP, we introduce a slacked version of the MinRess problem by replacing constraint (22) with:

$$
\sum_{d \in \mathcal{D}} \sum_{p \in P_{d}} \delta_{e d p} f_{d p}+s_{e}^{\prime \prime}=k_{e}^{\prime \prime} c_{e}, \quad \forall e \in \mathcal{E}
$$

Following the same reasoning as for ApproxMinLSP, we get:

$$
\mathrm{Nb} \text { of LSPs }+\mathrm{Nb} \text { of non-zero } s_{e}^{\prime \prime} \leq l+m
$$

Hence, increasing the number of non-zero $s_{e}^{\prime \prime}$ will reduce the number of LSPs and is equivalent to increasing $k_{e}^{\prime \prime}$ (or $\beta$ ) in constraint (22) of the original problem. Finally, MinLSP is an MILP with a polynomial number of paths and can be efficiently solved by commercial solvers using branch and bound techniques (though no guarantee for polynomial time complexity is given when using this optional component).

\section{LP 4 MinLSP}

$$
\text { Minimize } \sum_{d \in \mathcal{D}} \sum_{p \in \mathcal{P}_{d}^{\prime}} z_{d p}
$$

Subject to:

$$
\begin{gathered}
\sum_{d \in \mathcal{D}} \sum_{p \in \mathcal{P}_{d}^{\prime}} \delta_{e d p} f_{d p} \leq k_{e}^{\prime \prime} c_{e}, \quad \forall e \in \mathcal{E} \\
\sum_{p \in \mathcal{P}_{d}^{\prime}} f_{d p}=h_{d}, \quad \forall d \in \mathcal{D} \\
0 \leq f_{d p} \leq H z_{d p}, \quad \forall(d, p) \in \mathcal{D} \times \mathcal{P}_{d}^{\prime} \\
z_{d p} \in\{0,1\}, \quad \forall(d, p) \in \mathcal{D} \times \mathcal{P}_{d}^{\prime}
\end{gathered}
$$

$\mathbb{K}^{\prime \prime}=\beta \mathbb{K}^{\prime}$, where $\mathbb{K}^{\prime}$ is the congestion in MinRess

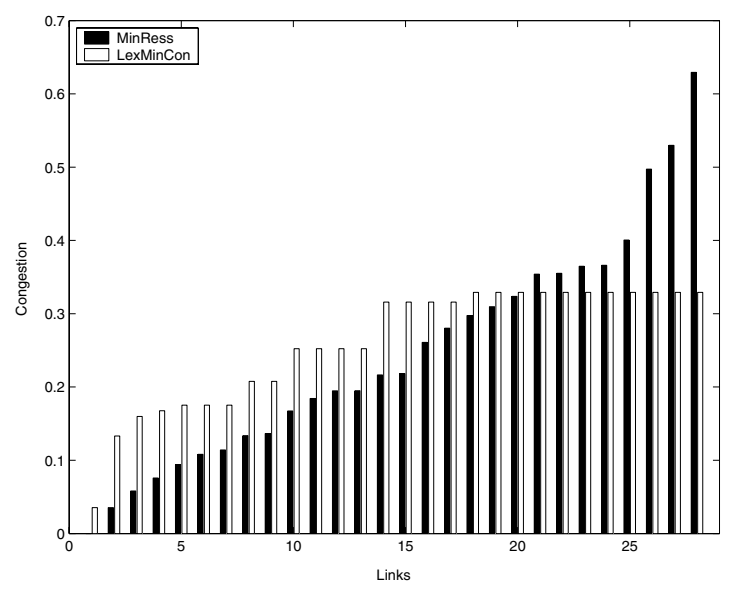

Fig. 2. Congestion state - Single objective approach (KL, $l=40)$

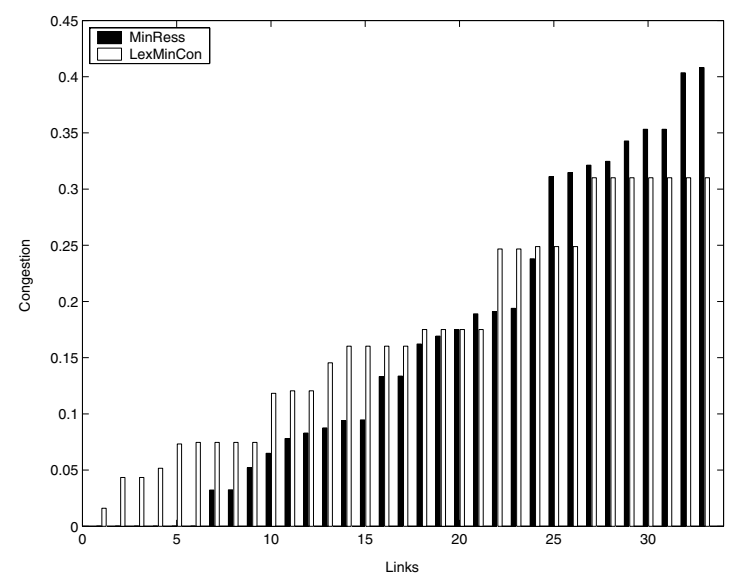

Fig. 3. Congestion state - Single objective approach (MCI, $l=30$ )

\section{Simulation AND Results}

In order to evaluate the performance of our approach, we perform a series of simulations on typical topologies used in the state of the art: the MCI network topology [10] (19 nodes, 33 links), the KL topology (15 nodes, 28 links), the REG topology (14 nodes, 29 links) and the NSF topology (8 nodes, 10 links) [6]. The linear programs were formulated with MATLAB and solved using an external interface (MEXfunction) to the lpsolve package [11]. In our simulation scenarios, the traffic demands are uni-directional and the bandwidth for each demand is drawn from a uniform distribution over the range of $[10,50]$ bandwidth units. The edge capacities are obtained from a uniform distribution over the range of $[250,650]$ bandwidth units.

In our first set of simulations, we show the relevancy of including both congestion and resource minimization components in our framework. Therefore, we run simulations considering each time a single objective optimization chosen between LexMinCon and MinRess. Figure 2 illustrates the congestion state for the KL network with 40 demands. We can clearly see that MinRess induces a highly congested network, while LexMinCon has the best performance with minimum congestion over all the network links. This is also valid for other simulation topologies and typical results are illustrated in 


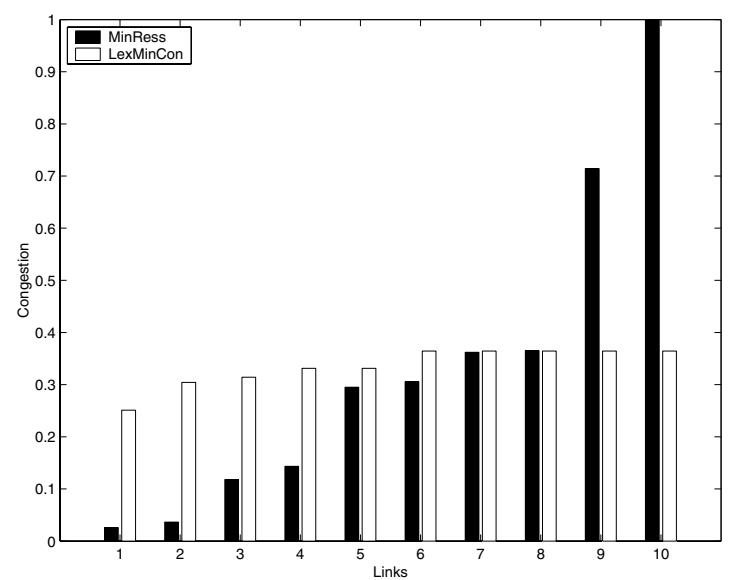

Fig. 4. Congestion state - Single objective approach (NSF, $l=25$ )

\begin{tabular}{l||c|c|c} 
& $\begin{array}{c}\text { MCI } \\
\end{array}$ & $\begin{array}{c}\text { KL } \\
l=30\end{array}$ & $\begin{array}{c}\text { NSF } \\
l=40\end{array}$ \\
\hline \hline MinRess & 2100 & 2600 & 1320 \\
\hline LexMinCon & 2400 & 3000 & 1590
\end{tabular}

TABLE I

BANDWIDTH CONSUMPTION - SINGLE OBJECTIVE APPROACH

Figure 3 and Figure 4. For instance, in the NSF case, MinRess leads to a highly congested network, with links approaching the maximal capacity, while LexMinCon distributes the load over the network links, thus reduces global congestion. However, if we observe the bandwidth consumption for each of the simulation runs, we can see in Table I that MinRess reaches the minimum bandwidth consumption, while LexMinCon induces a slightly higher bandwidth consumption. These typical results illustrate the inefficiency of approaches based on a single objective and the need for combining congestion and resource minimization as in the FATE framework.

Hence, our second set of simulations enables to evaluate the combination of the first two components in the FATE framework. Results for bandwidth consumption obtained after executing LexMinCon and MinRess successively are depicted in Table II. These results show that by increasing the value of $\alpha$ we can lower the bandwidth consumption. Moreover, we find it useful to compare these results with the performance of MinRess used as a single objective (Table III). For instance, considering the REG topology with 60 demands, we get a minimal resource consumption (3303 bandwidth units) by slightly increasing the congestion state $(\alpha=1.2)$.

Our final set of simulations provides insights on the total

\begin{tabular}{l||c|c|c|c}
\multicolumn{1}{c||}{} & \multicolumn{2}{c|}{ MCI } & \multicolumn{2}{c}{ REG } \\
\cline { 2 - 5 }$\alpha$ & $l=30$ & $l=50$ & $l=40$ & $l=60$ \\
\hline \hline 1 & 1888 & 3585 & 2249 & 3418 \\
\hline 1.2 & 1765 & 3404 & 2138 & 3303 \\
\hline 1.4 & 1749 & 3404 & 2119 & 3303 \\
\hline 1.8 & 1744 & 3404 & 2102 & 3303 \\
\multicolumn{3}{c}{ TABLE II }
\end{tabular}

BANDWIDTH CONSUMPTION - FATE: OUTPUT OF THE MinRess COMPONENT

\begin{tabular}{c||c|c|c|c}
\multicolumn{1}{c||}{} & \multicolumn{2}{c|}{ MCI } & \multicolumn{2}{c}{ REG } \\
\cline { 2 - 5 } & $l=30$ & $l=50$ & $l=40$ & $l=60$ \\
\hline \hline MinRess & 1744 & 3404 & 2102 & 3303 \\
& \multicolumn{3}{c}{ TABLE III } &
\end{tabular}

BANDWIDTh CONSUMPTION - Single obJective MinRess

\begin{tabular}{l||c|c|c|c|c} 
& \multirow{2}{*}{$\begin{array}{c}\text { Min- } \\
\alpha\end{array}$} & Approx- & \multicolumn{4}{|c}{ MinLSP } \\
\cline { 4 - 6 } & Ress & MinLSP & $\beta=1$ & $\beta=1.1$ & $\beta=1.2$ \\
\hline \hline 1 & 59 & 59 & 59 & 45 & 41 \\
\hline 1.2 & 51 & 45 & 44 & 41 & 41 \\
\hline 1.4 & 47 & 45 & 43 & 41 & 40 \\
\hline 1.8 & 43 & 42 & 40 & 40 & 40
\end{tabular}

TABLE IV

TOTAL NUMBER OF PATHS - FATE: OUTPUT OF EACH COMPONENT (MCI,

$$
l=40)
$$

number of paths in our framework. The first two columns of Table IV and Table V illustrate the number of paths obtained at the output of the MinRess and the ApproxMinLSP components for the MCI and REG topologies (with 40 and 30 demands respectively). We note that when $\alpha$ increases, the number of paths is reduced. However, there is no significant difference between the two values for MinRess and ApproxMinLSP since we find a BFS in both cases, thus the number of paths is already reduced for MinRess. Moreover, we note that this number is lower than $l+m$, validating the theoretical bound. In the last three columns of Table IV and Table V, we evaluate the performance of the optional MinLSP component for the same simulation cases. For instance, in the MCI case, we obtain an unsplittable flow allocation solution for $\beta=1.2$ and $\alpha=1.4$. Finally, special attention is given to the MinLSP solution procedure. In fact, this MILP has a polynomial size (due to the reduced path set) but the optimal solution can still be hard to compute, especially for lower values of $\alpha$ and $\beta$. Therefore, we choose a gap of $10^{-4}$ as the tolerance of the branch and bound algorithm (this gap is the difference between the best-found solution and the current solution) and an LU decomposition procedure as a basic factorization [11].

\section{CONCLUSION}

In this paper, we present FATE, a novel polynomial time framework for the flow allocation problem in MPLS-TE networks. FATE includes three tunable components that enable to successively reduce congestion, bandwidth consumption and the number of LSPs in use. The solution obtained with FATE is an $\left(\alpha, 1+\frac{m}{l}\right)$-approximation for the general flow allocation

\begin{tabular}{l||c|c|c|c|c} 
& \multirow{2}{*}{$\begin{array}{c}\text { Min- } \\
\alpha\end{array}$} & Rpprox- & \multicolumn{3}{|c}{ MinLSP } \\
\cline { 4 - 6 } & MinLSP & $\beta=1$ & $\beta=1.1$ & $\beta=1.2$ \\
\hline \hline 1 & 46 & 46 & 46 & 35 & 33 \\
\hline 1.2 & 41 & 37 & 34 & 33 & 31 \\
\hline 1.4 & 37 & 34 & 32 & 30 & 30 \\
\hline 1.8 & 34 & 31 & 30 & 30 & 30 \\
\multicolumn{3}{|c|}{ TABLE V }
\end{tabular}

TOTAL NUMBER OF PATHS - FATE: OUTPUT OF EACH COMPONENT (REG, $l=30)$ 
problem. Simulation results that are presented in this work provide concrete proof of the importance of each component and insights on the tuning process. Moreover, we show that the flow allocation solution can be obtained in a polynomial time so results can be further extended to larger topologies with a big number of demands. Our future work will study a possible extension for the FATE framework that enables to find efficient flow allocation solutions for survivable flows with end-to-end restoration requirements.

\section{APPENDIX}

LexMinCon, formulated in LP 1, can be solved in a polynomial time as a sequence of mathematical problems following Algorithm 1, introduced in [12]. At each iteration of this

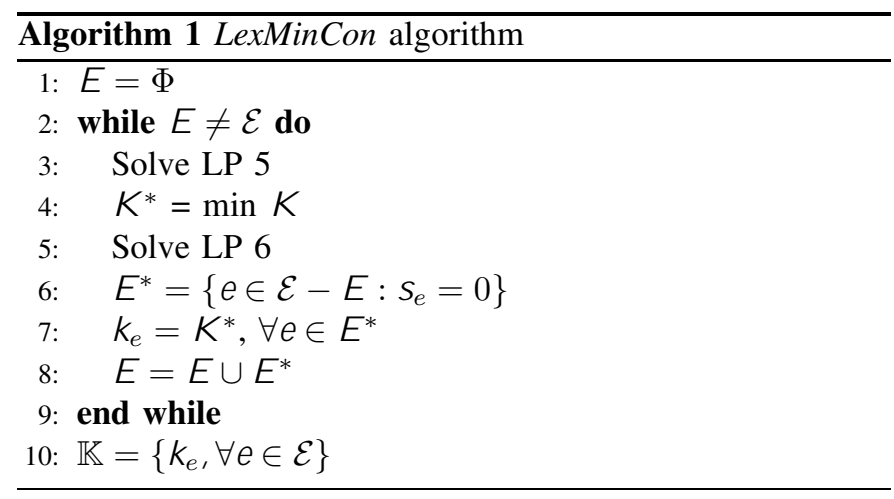

algorithm, $E$ captures the subset of $\mathcal{E}$ including links that has the congestion fixed to the final value (as it will be in the output vector $\mathbb{K}$ ). First, we solve an elementary problem formulated in LP 5 and get a value of $K^{*}$. Then, we need to

LP 5 Elementary problem

$$
\text { Minimize } K
$$

Subject to:

$$
\begin{gathered}
\sum_{d \in \mathcal{D}} f_{d e} \leq K c_{e}, \forall e \in \mathcal{E}-E \\
\sum_{d \in \mathcal{D}} f_{d e}=k_{e} c_{e}, \quad \forall e \in E \\
\sum_{e \in \operatorname{Out}(v)} f_{d e}-\sum_{e \in \operatorname{In}(v)} f_{d e}=\epsilon_{d v}, \quad \forall(d, v) \in \mathcal{D} \times \mathcal{V} \\
f_{d e} \geq 0, \quad \forall(d, e) \in \mathcal{D} \times \mathcal{E} \\
K \geq 0
\end{gathered}
$$

find the set of binding constraints in this elementary problem; binding constraints are defined as the subset of constraints that verify an equality relation in (28) and correspond to the set of links $E^{*}$ given by:

$$
E^{*}=\left\{e \in \mathcal{E}-E: \sum_{d \in \mathcal{D}} f_{d e}=K^{*} c_{e}\right\} .
$$

Practically, in order to get $E^{*}$, we solve a slacked version of the problem, formulated in LP 6 , using additional slack variables $s_{e}$. Now the binding constraint set is given by:

$$
E^{*}=\left\{e \in \mathcal{E}-E: s_{e}=0\right\} .
$$

Therefore, for all the links in $E^{*}, k_{e}$ takes a final congestion value of $K^{*}$ and these links are added to the set $E$. After iterating over the set $E$, we finally get $\mathbb{K}$, the lexicographically minimal vector with all its elements given by the final $k_{e}$ values.

LP 6 Slacked problem

$$
\text { Maximize } \sum_{e} s_{e}
$$

Subject to:

$$
\begin{gathered}
\sum_{d \in \mathcal{D}} f_{d e}+s_{e} \leq K^{*} c_{e}, \quad \forall e \in \mathcal{E}-E \\
\sum_{d \in \mathcal{D}} f_{d e}=k_{e} c_{e}, \quad \forall e \in E \\
\sum_{e \in \text { Out }(v)} f_{d e}-\sum_{e \in \operatorname{In}(v)} f_{d e}=\epsilon_{d v}, \quad \forall(d, v) \in \mathcal{D} \times \mathcal{V} \\
f_{d e} \geq 0, \quad \forall(d, e) \in \mathcal{D} \times \mathcal{E} \\
s_{e} \geq 0, \quad \forall e \in \mathcal{E}-E
\end{gathered}
$$

The polynomiality of LexMinCon follows from the fact that Algorithm 1 computes the optimal lexicographically minimum load vector in at most $(2 m-1)$ LP computations and each of them can be solved in a polynomial time [8]. Note that more sophisticated approaches can be used for this problem, as it is strictly related to the max-min fairness optimization in [1].

\section{REFERENCES}

[1] M. Pióro and D. Medhi, Routing, Flow and Capacity Design in Communication and Computer Networks. Morgan Kaufmann Publishers (Elsevier), June 2004.

[2] S. C. Erbas and C. Erbas, "A multiobjective off-line routing model for mpls networks," in Proc. of the 18th International Teletraffic Congress, Aug.-Sep. 2003.

[3] S. Shekhar Srivastava and D. Medhi, "Network traffic engineering with varied levels of protection in the next generation internet," Performance Evaluations and Planning Methods for the Next Generation Internet Februrary 2005.

[4] Y. Lee et al., "A constrained multipath traffic engineering scheme for mpls networks," in ICC, April 2002.

[5] C. T. Chou, "Traffic engineering for mpls-based virtual private networks," Computer Networks, vol. 44, no. 3, pp. 319-333, February 2004.

[6] V. S. Mirrokni et al., "A simple polynomial time framework for reducedpath decomposition in multi-path routing," in IEEE INFOCOM, 2004.

[7] A. Chakrabarti et al., "Approximation algorithms for the unsplittable flow problem," in 5th International Workshop on Approximation Algorithms for Combinatorial Optimization, 2002.

[8] E. Tardos, "A strongly polynomial algorithm to solve combinatorial linear programs," Operations Research, vol. 34, 1986.

[9] T. Ahuja, R. Magnanti and J. B. Orlin, Network flows: theory, algorithms, and applications. Upper Saddle River, NJ: Prentice-Hall, Inc., 1993.

[10] S. Nelakuditi and Z. Zhang, "On selection of paths for multi-path routing," in IWQoS, June 2001.

[11] K. Berkelaar, M. Eikland and P. Notebaert, "Open source (mixed-integer) linear programming system," Version 5.1.0.0 - GNU LGPL (Lesser General Public Licence), May 2004

[12] D. Nace and J. B. Orlin, "Lexicographically minimum and maximum load linear programming problems," http://web.mit.edu/jorlin/www/papersfolder/LexMinLoad.pdf, August 2004. 